\title{
Turbinas Eólicas: Optimización en el Pre-Diagnóstico de Fallas en el Generador
}

\author{
Horacio R. di Prátula, Eduardo Guillermo, Andrea P. Rossi y Rodolfo Bocero \\ Universidad Tecnológica Nacional, Facultad Regional Bahía Blanca, Depto. de Ingeniería \\ Eléctrica, Grupo de Estudios Sobre Energía, 11 de Abril 461, B8000LMI Bahía Blanca, \\ Buenos Aires-Argentina. (email: hrdiprat@bblanca.com.ar, eguiller@frbb.utn.edu.ar, \\ aprossi@frbb.utn.edu.ar, rbocero@bblanca.com.ar \\ * Autor a quien debe ser dirigida la correspondencia
}

Recibido Jun. 30, 2011; Aceptado Ago. 05, 2011; Versión Final recibida Sep. 26, 2011

\begin{abstract}
Resumen
Se propone un método no invasivo y de bajo costo para el pre-diagnóstico de fallas en a máquina eléctrica de grandes aerogeneradores. Esto con el objeto de minimizar el riesgo de falla y la remoción de la máquina con uso de equipo pesado (grúas) para reparaciones. Dado que es usual recurrir a más de un método para detectar fallas incipientes, la predicción requiere corroboración para mayor seguridad. En la industria, esta práctica es normal (medir vibraciones y espectro de corriente, por ejemplo), pero optimizar el mantenimiento predictivo de una máquina eléctrica fijada en una turbina eólica requiere de la menor cantidad posible de dispositivos y de la factibilidad de utilizar monitoreo a distancia con métodos no invasivos. El método propuesto reúne estas condiciones y los ensayos preliminares con sensores de bajo costo muestran buenos resultados para su aplicación experimental.
\end{abstract}

Palabras clave: aerogenerador, diagnóstico de fallas, mantenimiento, método no invasivo, máquina eléctrica.

\section{Wind Turbine: Optimization of Failures Pre-Diagnosis in the Power Generator}

\begin{abstract}
A non-invasive low cost method for pre-diagnosis faults in electrical machines of large wind turbines is proposed. The objective is to minimize fault risks and the removal of the machine with heavy equipment (such as cranes) for maintenance. Since it is normal to use more than one method to detect incipient faults, prediction requires corroboration for higher security (such as vibration measurement and current spectrum analyses). This practice is usual in industries, but optimum predictive maintenance of electric machines in a wind turbine requires the use of the fewest possible devices and a distance non-invasive monitoring method. The proposed method has all this characteristics and preliminary essays with low cost sensors show good results for experimental applications.
\end{abstract}

Keywords: wind turbine, failures diagnosis, maintenance, non-invasive method, electric machine. 


\section{INTRODUCCIÓN}

Tanto la operación como el mantenimiento de las turbinas eólicas tienen gran incidencia en la rentabilidad de los parques eólicos (Walford, 2006), y por dicho motivo deben tenerse en cuenta al realizar la evaluación de dicha rentabilidad. En este sentido, resulta muy conveniente el diagnóstico precoz de ciertas fallas que producen costos significativos en la reparación (que incluye el uso de grúa y transporte) y un gran lucro cesante, a menos que se cuente con generadores en stock.

La tendencia es apuntar a un menor costo en operación y mantenimiento, y a una mayor disponibilidad del parque. En granjas eólicas en tierra (onshore) la disponibilidad debe superar el 97\% mientras que en mar (offshore) la disponibilidad varía del 80 al 95\% (reflejo de lo incipiente de su tecnología) (Henderson et al., 2009).

El presente trabajo propone efectuar un pre-diagnóstico de fallas en la máquina eléctrica, censando el campo magnético disperso sobre la misma, y evaluando la variación del autovalor de la matriz que resulta del producto entre la matriz de flujo y su transpuesta. El método se basa en expresar mediante matrices la estructura física de la máquina eléctrica, discretizando tanto el bobinado como el circuito magnético de la misma (Bambill, 1989; di Prátula, 2003a). Ante una falla, el autovalor de la matriz conformada con valores medidos del campo magnético externo comenzará a variar antes de que la falla pueda ser diagnosticada por los métodos habituales.

El desarrollo teórico matricial y la metodología experimental del método de pre-diagnóstico aquí propuesto, se presentó por primera vez en la tesis doctoral de di Prátula (2008), cuya realización fue precedida de una búsqueda exhaustiva de publicaciones nacionales e internacionales de los últimos cuarenta años en la ISI-WEB de Science, y en bases de datos del IEEE y de otras sociedades científicas, para constatar y demostrar la originalidad del método.

La configuración geométrica de cada parte de la máquina, y la consecuente interrelación energética entre las mismas, determina el comportamiento eléctrico y mecánico de la máquina eléctrica. Cualquier modificación de la configuración energética existente implica el comienzo de una falla, por lo que el diagnóstico se realiza a través del flujo que es la manifestación directa de ambos sistemas: el eléctrico y el magnético (di Prátula, 2003b).

Además, dado que todas las fallas modifican la estructura física de la máquina (fallas de bobinados, rodamientos, desalineación, desbalanceo, etc.), excepto aquellas que dependen de las condiciones de conexión con la red eléctrica, el método aquí propuesto permite evaluar e identificar dichos problemas también.

Según Henao et al. (2003), el flujo disperso contiene las frecuencias de falla. Por lo tanto, si se le realiza al mismo un espectro de frecuencia, se obtendrán las frecuencias de falla y se podrán diagnosticar tanto fallas mecánicas como eléctricas (di Prátula, 2009).

Dado que el flujo total surge de la acción de dos fuerzas magnetomotrices debido a la corriente que circula por los bobinados estatórico y rotórico, uno estacionario y otro móvil, sobre el circuito magnético principal de conversión, si se considera el entrehierro (de longitud radial $\delta$ y diámetro medio $D_{m}$ ) con mayor reluctancia que el resto del circuito magnético (ec. 1), se puede suponer la linealidad del flujo respecto a la fuerza magnetomotriz de excitación. La ecuación (1) muestra la expresión de la permeancia de una máquina eléctrica.

$$
\mathbf{P}=\frac{\mu_{0} A}{\delta}=\frac{4 \pi 10^{-7} \pi D_{m} \ell}{\delta} \quad[H]
$$

El análisis del flujo total en el circuito magnético principal de la máquina contendrá las frecuencias de falla y, tal como demuestra Henao et al. (2003), las mismas estarán presentes en el flujo disperso. Esto significa que tanto fallas mecánicas como eléctricas podrán diagnosticarse 
mediante la medición externa de flujo. De este modo se propone que, mediante la medición del flujo con sensores con núcleo de aire, tanto en sentido axial de la máquina eléctrica como en sentido perpendicular (perimetral), configurando una matriz de valores tomados en puntos fijos, se obtengan valores de flujo conformando una matriz de valores durante el tiempo de medición.

Sin embargo, las fallas estatóricas no muestran frecuencias de falla particulares sino modificación de la energía (manifestación razonable ante una menor inductancia propia y mutua con el resto de las bobinas), y es así que se demuestra que la matriz resultante del producto de la matriz de flujo por su transpuesta tiene autovalores que varían con ese tipo de falla.

\section{ANÁLISIS Y CONTRIBUCIÓN TEÓRICA}

El método de diagnóstico experimental y el análisis teórico se basan en un planteo matricial de las estructuras eléctricas y magnéticas de la máquina eléctrica. Dicho planteo considera la matriz densidad numérica de conductores $\mathbf{Z}_{\mathrm{Q}}(\mathrm{qxm})$ del circuito, siendo "q" el número de ranuras y " $\mathrm{m}$ " el número de fases del bobinado. Cada elemento $Z_{Q_{i, j}}$ corresponde a la suma de los impulsos de conductores despreciando el ancho de ranura (Fig. 1a).

La función densidad de conductores $\mathrm{N}(\alpha)$ se define como la suma de funciones componentes sucesivas, correspondiente cada una a un pulso espacial de valor constante $\mathrm{Z}_{\mathrm{Q}} / \mathrm{X}_{\mathrm{C}}$, correspondiendo a un pulso solo en una franja (ranura) y nulo fuera de él, de valor medio nulo. $O$ también se la define como una función impulso (caso límite del anterior donde $X_{C}$ es despreciado) de densidad de conductores de valor $\mathrm{Z}_{\mathrm{Q}}$ en una posición angular correspondiente a la ranura, siendo " $\alpha$ " dicha posición angular.

$N(\alpha)=\sum N(\alpha)=d \mu(\alpha) / d \alpha$

Entonces, $\mathrm{N}(\alpha)$ es una función pulso o impulso definida en una posición angular determinada, y $\mathrm{Z}_{\mathrm{Q}}$ es el número de conductores por ranura (sumados con el signo correspondiente al sentido de corriente por cada uno), siendo $\mathrm{X}_{\mathrm{C}}$ el arco correspondiente al ancho de ranura.

A partir de esta función, y como consecuencia la matriz densidad de conductores, se define la matriz bobinado como una discretización de la función bobinado (enlace) en el recinto de la máquina eléctrica.

La función enlace $\mu(\alpha)$ (Bambill, 1989; di Prátula, 2003a) queda definida como la integral de la densidad de conductores determinada por hacer nulo el valor medio del flujo específico $\mathrm{b}(\alpha)=\mathrm{fmm}(\alpha) \mathrm{P}(\alpha)=\mathrm{b}(\alpha \pm 2 \pi)$ inducido por el circuito. La ecuación (3) expresa claramente la idea.

$$
\mu\left(\alpha, \alpha \pm \alpha_{p / 2}\right)=\sum_{\alpha}^{\alpha \pm \alpha_{p} / 2} N_{\alpha}(\alpha)=\int_{\alpha p / 2} N(\alpha) d \alpha
$$

De este modo la matriz bobinado $\mathbf{N}(\chi \times \mathrm{m})$ :

$\mathbf{N}(\chi \times m) \in \mathrm{R}^{\chi \times \mathrm{m}}$

donde " $\chi$ " es el número de posiciones angulares espaciales fijas adoptadas de la máquina eléctrica y "m" las fases (Fig. 1).

En síntesis, cada columna de la matriz corresponde a una fase, y cada fila "establece" una posición angular en el entrehierro de la máquina eléctrica con respecto a un eje de referencia de 
posición arbitraria. La matriz bobinado (Xiaogang et al., 1995; Al-Nuaim y Toliyat, 1998) ha surgido de la matriz densidad de conductores, pero también puede deducirse a partir de la función bobinado, discretizando la misma.

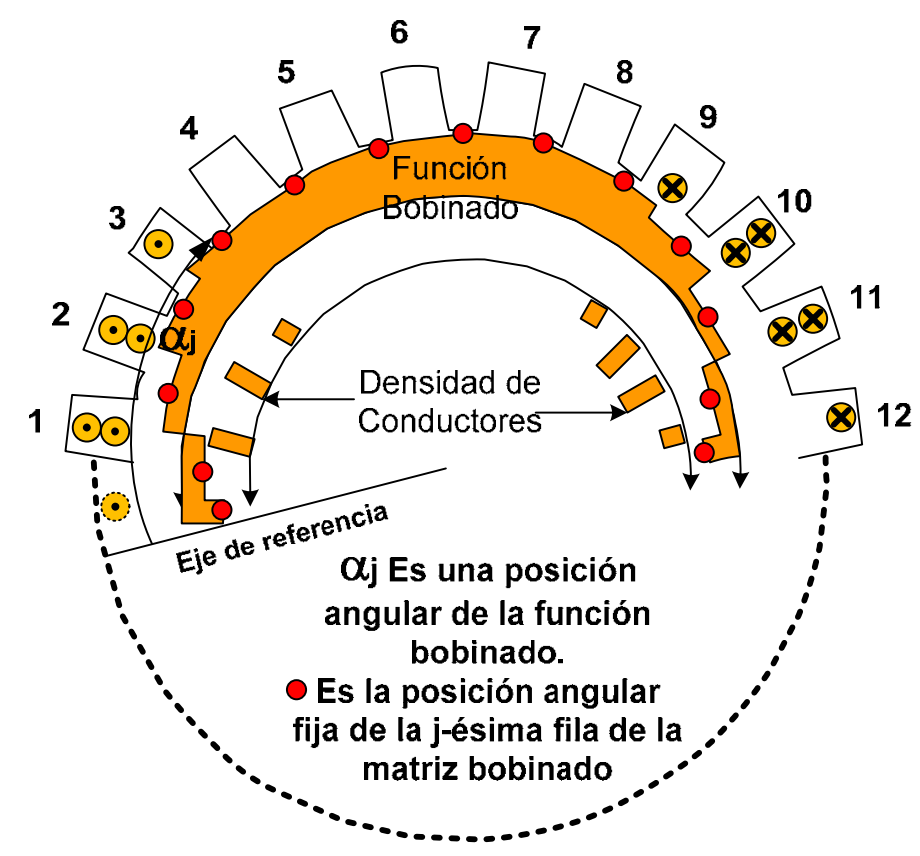

Fig. 1: Máquina eléctrica, bobinado estatórico, función bobinado (enlace) y posiciones angulares fijas (di Prátula, 2003b)

A partir de esta definición matricial de la estructura eléctrica (bobinado) de la máquina, puede definirse la estructura magnética. Sus filas determinan su magnitud en cada posición angular fija adoptada (matriz bobinado), y sus columnas, la magnitud a lo largo del entrehierro en cada instante de tiempo (se definen de acuerdo a la ecuación (3) en función de la velocidad de rotación del rotor) (Fig. 2).

Un análisis espectral de la fila y de la columna de la matriz permitiría conocer su variación en el tiempo para cada posición angular fija en el entrehierro, y la variación espacial de la permeancia a lo largo del entrehierro para un instante de tiempo, respectivamente.

La matriz bobinado $\mathbf{I}(\chi \times \mathrm{m})$ multiplicada por la corriente de excitación $\mathbf{I}\left(\mathrm{m} \times \omega_{\mathrm{n}}\right)$ determina la fuerza magnetomotriz $\mathbf{F}\left(\chi \times \omega t_{n}\right)$ producida por el bobinado considerado (ec. 5).

$\mathbf{F}\left(\chi \times \omega \mathrm{t}_{\mathrm{n}}\right) \in \mathrm{R}^{\chi \times \mathrm{m}}$, siendo $\mathbf{F}\left(\chi \times \omega \mathrm{t}_{\mathrm{n}}\right)=\mathbf{I}(\chi \times \mathrm{m}) * \mathbf{I}\left(\mathrm{m} \times \omega \mathrm{t}_{\mathrm{n}}\right)$ la fuerza magnetomotriz

$\mathbf{I}(\chi \times \mathrm{m}) \in \mathrm{R} \chi \times \mathrm{m}$, siendo $\mathbf{I}=\left[\mathbf{i}_{\omega \mathrm{t}_{1}} \cdots \cdots \mathbf{i}_{\omega \mathrm{t}_{\mathrm{n}}}\right]=\left[\begin{array}{c}\mathbf{i}_{1} \\ \vdots \\ \mathbf{i}_{2}\end{array}\right]$ la corriente en un bobinado

El flujo total de la máquina eléctrica (ec. 6) se producirá debido a la fuerza magnetomotriz de excitación, actuando en el circuito magnético cuya permeancia está representada por la matriz de la ecuación (7), cuyas filas representan la permeancia en cada posición angular, y las columnas, instantes de tiempo.

$$
\begin{aligned}
& \Phi\left(\chi \times \omega \mathrm{t}_{\mathrm{n}}\right)=\left(\overline{\mathbf{S F}\left(\chi \times \omega \mathrm{t}_{\mathrm{n}}\right) * \mathbf{P}\left(\chi \times \omega \mathrm{t}_{\mathrm{n}}\right)}\right) \\
& \Phi\left(\chi \times \omega \mathrm{t}_{\mathrm{n}}\right) \in \mathrm{R}^{\chi \times \omega \mathrm{t}_{\mathrm{n}}}
\end{aligned}
$$


$\mathbf{S F}\left(\chi \times \omega \mathrm{t}_{\mathrm{n}}\right) \in \mathrm{R}^{\chi \times \mathrm{m}}$ representa la suma de las fuerzas magnetomotrices actuando en la geometría espacial de conversión de la máquina eléctrica $\left(\mathbf{S F}_{\mathbf{i j}}\left(\chi \times \omega \mathrm{t}_{\mathrm{n}}\right)=\mathbf{F}_{\mathbf{i}}\left(\chi \times \omega \mathrm{t}_{\mathrm{n}}\right)+\mathbf{F}_{\mathbf{j}}\left(\chi \times \omega \mathrm{t}_{\mathrm{n}}\right)\right)$, originadas en las estructuras eléctricas excitadas $\mathrm{e}$ interrelacionadas entre sí.

$\mathbf{P}(\chi \times \mathrm{m}) \in \mathrm{R} \chi \times \mathrm{m}$, siendo $\mathbf{P}=\left[\mathbf{p}_{\omega \mathrm{t}_{1}} \cdots \cdots \mathbf{p}_{\omega \mathrm{t}_{\mathrm{n}}}\right]=\left[\begin{array}{c}\mathbf{p}_{1} \\ \vdots \\ \mathbf{p}_{2}\end{array}\right]$

El flujo de dispersión es producido por las mismas estructuras, aunque la geometría espacial no es la de conversión y la matriz de permeancia es otra. Es por eso que se puede afirmar que las frecuencias componentes existen tanto en el flujo principal como en el de dispersión (Henao et al., 2003).

La matriz del flujo disperso medido sobre la máquina eléctrica en diferentes lugares queda representado por la ecuación (8), donde las filas representan los valores de los sensores axiales y corresponden a posiciones angulares fijas en la máquina, y las columnas indican los valores de los sensores dispuestos a lo largo del perímetro de la máquina para diferentes posiciones angulares de la máquina (Fig. 2).

$$
\mathbf{B}=\left(\begin{array}{llll}
\Phi_{11} & \Phi_{12} & \Phi_{13} & \Phi_{14} \\
\Phi_{21} & \Phi_{22} & \Phi_{23} & \Phi_{24} \\
\Phi_{31} & \Phi_{32} & \Phi_{33} & \Phi_{34} \\
\Phi_{41} & \Phi_{42} & \Phi_{43} & \Phi_{44} \\
\Phi_{51} & \Phi_{52} & \Phi_{53} & \Phi_{54}
\end{array}\right)
$$

Los elementos de la matriz de la ecuación (8) se calculan con la ecuación (9) para las columnas, y la ecuación (10) para las filas.

$$
\begin{aligned}
& \Phi_{11}=\frac{\mathbf{N} \mathbf{i}_{1} k}{\delta_{1}}, \Phi_{21}=\frac{\mathbf{N} \mathbf{i}_{1} k}{\delta_{1}}, \ldots, \Phi_{51}=\frac{\mathbf{N} \mathbf{i}_{1} k}{\delta_{1}} \\
& \Phi_{11}=\frac{\mathbf{N} \mathbf{i}_{1} k}{\delta_{1}}, \Phi_{12}=\frac{\mathbf{N} \mathbf{i}_{2} k}{\delta_{2}}, \ldots, \Phi_{14}=\frac{\mathbf{N} \mathbf{i}_{4} k}{\delta_{4}}
\end{aligned}
$$

Ante una falla, el rotor verá afectado su entrehierro al girar, el flujo será modulado por esa variación y afectará los valores $\delta_{1}, \delta_{2}, \delta_{3}, \delta_{4}, \delta_{5}$ de los sensores a lo largo del perímetro de la máquina eléctrica. Multiplicando la matriz $\mathbf{B}$ por su traspuesta, se determinan los autovalores $\left[\mathrm{B}(\chi \times\right.$ tpo $) * \mathrm{~B}^{\mathrm{T}}($ tpo $\left.\mathrm{x} \chi)=\left(\mathrm{B}^{*} \mathrm{~B}^{\mathrm{T}}\right)(\chi \times \chi)\right]$ que están relacionados con la energía de dispersión en cada posición angular (ec.11). La ecuación (11) muestra que existe un autovalor fuertemente influenciado por las variaciones del entrehierro y, por lo tanto, la variación está relacionada a fallas mecánicas.

$$
\text { autovalores }\left(\mathbf{B} * \mathbf{B}^{\mathbf{T}}\right) \rightarrow\left[\begin{array}{c}
0 \\
0 \\
0 \\
0 \\
5 \mathbf{i}_{1}^{2} \mathbf{N}^{2} \mathbf{k}^{2} \delta_{2}^{2} \delta_{3}^{2} \delta_{4}^{2}+5 \mathbf{i}_{2}^{2} \mathbf{N}^{2} \mathbf{k}^{2} \delta_{1}^{2} \delta_{3}^{2} \delta_{4}^{2}+5 \mathbf{i}_{3}^{2} \mathbf{N}^{2} \mathbf{k}^{2} \delta_{1}^{2} \delta_{2}^{2} \delta_{4}^{2}+5 \mathbf{i}_{4}^{2} \mathbf{N}^{2} \mathbf{k}^{2} \delta_{1}^{2} \delta_{2}^{2} \delta_{3}^{2} \\
\hline \delta_{1}^{2} \delta_{2}^{2} \delta_{3}^{2} \delta_{4}^{2}
\end{array}\right]
$$


Si se asocia el valor de $\delta$ con una variación senoidal (Riley et al., 1997) y se lo reemplaza en la matriz de flujo, los autovalores quedan expresados por la ecuación (12), siendo $\delta=\operatorname{sen}(\mathrm{z})-$ variación periódica del entrehierro producido por vibración externa.

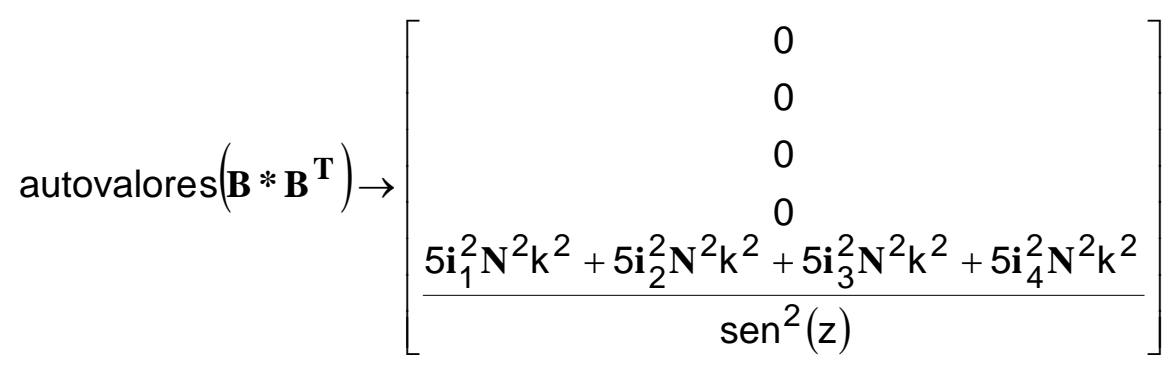

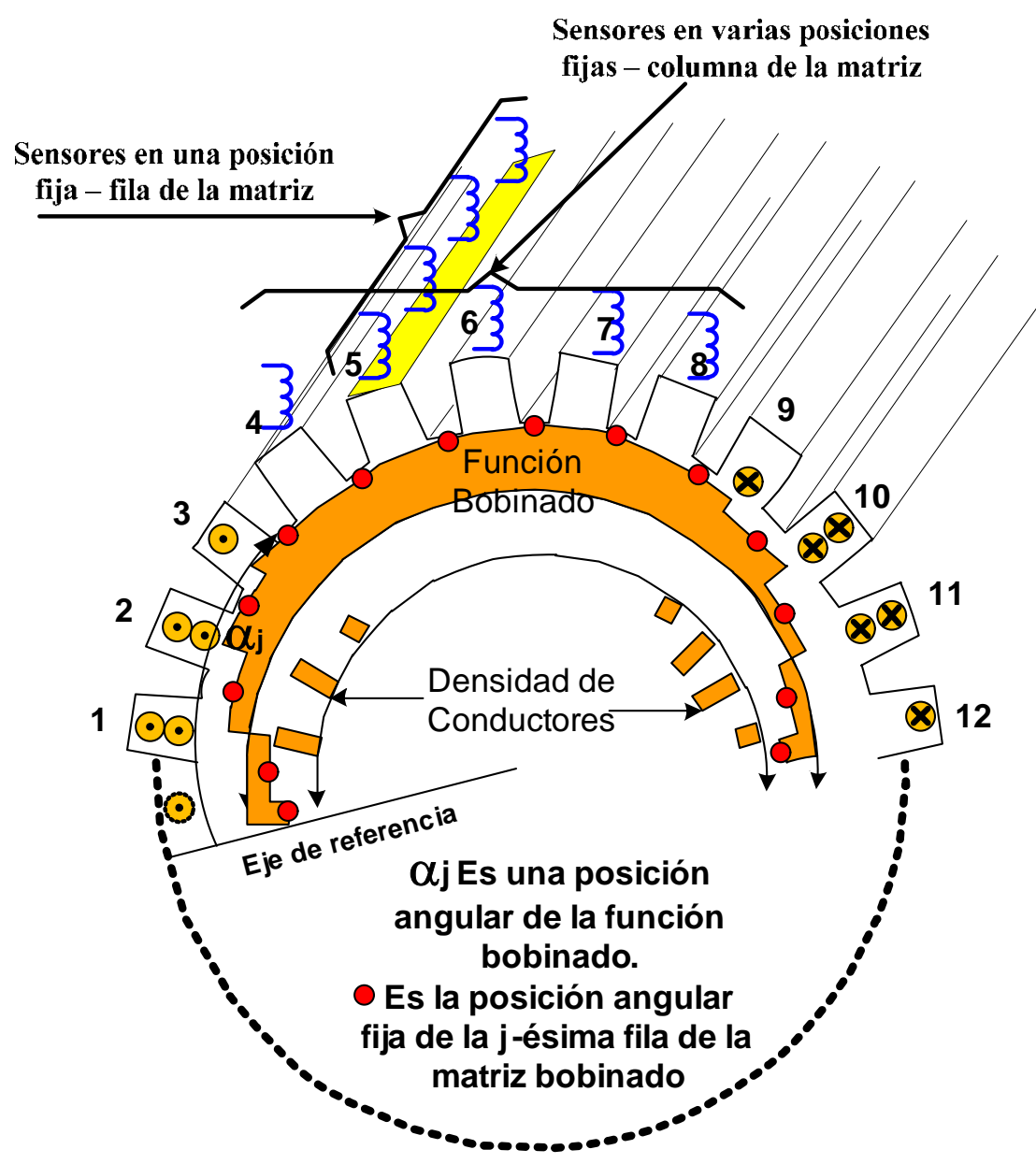

Fig. 2: Posición de sensores sobre la máquina eléctrica.

Si se analizan las condiciones de funcionamiento en falla de acuerdo a lo expresado en Henao et al. (2003), el flujo de dispersión contiene las frecuencias de falla del rotor (ec. 13), y para la falla estatórica modificará el valor de los autovalores (ec. 14) (se considera que la variación del autovalor indica una variación en los enlaces magnéticos entre bobinas).

Una falla mecánica (como por ejemplo: una excentricidad dinámica y estática que modifica la estructura magnética) puede caracterizarse por una variación de entrehierro, y presentaría una ecuación de flujo como la ecuación (13) (Fernandez Cabanas et al., 1998; Schoen et al., 1995; Dorrell et al., 1997). 


$$
\Phi_{\mathrm{d}}=\left(\frac{\mathrm{k} \mathbf{I} \mathrm{I}_{\mathrm{C}}}{\mathbf{p}^{2} \delta}\right) \operatorname{sen}[1-v(1-\mathrm{s}) \omega \mathrm{t}-\mathrm{k} v]+\left(\frac{\mathrm{k} \mathbf{I} \mathrm{I}_{\mathrm{C}}}{\gamma \delta}\right) \operatorname{sen}\left[\gamma \frac{\mathrm{s} \omega \mathrm{t}}{\mathbf{p}}+\mathrm{k} \gamma\right]
$$

$\mathbf{T} \rightarrow\left[\begin{array}{c}0 \\ 0 \\ 0 \\ 0 \\ 20 \gamma^{2} \mathbf{I}^{2} k^{2} I_{\mathrm{c}}^{2} \operatorname{sen}^{2}(\omega \mathrm{stv}-\omega \mathrm{tv}-\mathrm{kv}+1)+40 \gamma^{2} \mathbf{I} \mathbf{I}_{\mathrm{r}} \mathrm{k}^{2} \mathrm{I}_{\mathrm{C}}^{2} \mathbf{p} \operatorname{sen}(\omega \operatorname{stv}-\omega \mathrm{tv}-\mathrm{kv}+1) \operatorname{sen}\left(\frac{\gamma \mathbf{k} \mathbf{p}+\gamma \omega \mathbf{s t}}{\mathbf{p}}\right)+20 \mathbf{I}_{\mathrm{r}}^{2} \mathrm{k}^{2} \mathrm{I}_{\mathrm{c}}^{2} \mathbf{p}^{4} \operatorname{sen}^{2}\left(\frac{\gamma \mathbf{k} \mathbf{p}+\gamma \omega \mathbf{s t}}{\mathbf{p}}\right) \\ \gamma^{2} \delta^{2} \mathbf{p}^{4}\end{array}\right]$

De acuerdo a la ecuación (14), en todos los casos el autovalor será afectado por las frecuencias de falla. De esta manera, las perspectivas de aplicación de este análisis matemático son las de establecer la condición de falla, y debido a que no la diagnostica, el mismo puede usarse como pre-diagnóstico de su existencia

Además, el método de medición propuesto y la teoría permiten, no sólo el pre-diagnóstico on-line, sino también el diagnóstico de falla a través de la aplicación de la Transformada Rápida de Fourier a filas y columnas de la matriz de flujo, y a la matriz columna conformada por las columnas de la matriz de flujo presentada.

Con el fin de ilustrar el análisis de los párrafos anteriores, se presenta el siguiente ejemplo teórico: en una máquina de inducción de cuatro polos, 36 ranuras y 20 conductores por ranura se considera una variación de entrehierro producida por una excentricidad dinámica y una estática. La permeancia queda expresada por la ecuación (15) (Fernandez Cabanas et al., 1998; Schoen et al., 1995; Dorrell et al., 1997).

$$
\mathbf{P}\left(\chi \times \omega \mathrm{t}_{\mathrm{n}}\right)=\left[\mathbf{p}_{1} \cdots \mathbf{p}_{\omega \mathrm{t}_{\mathrm{n}}}\right]=\mu_{0} \mathrm{~A}\left\{\delta_{0}^{-1}\left[1+\delta_{\mathrm{s}} \cos \left(\mathrm{p}^{-1} \alpha\right)+\delta_{\mathrm{d}} \cos \left((1-\mathrm{s}) \mathrm{p}^{-1} \omega \mathrm{t}-\mathrm{p}^{-1} \alpha\right)\right]\right\}
$$

En la ecuación (5) "s" es el resbalamiento, y la magnitud de la variación está determinada por " $\delta 0$ " que es el valor de la altura del entrehierro medio, y por " $\delta s$ " y " $\delta \mathrm{d}$ " que son valores tales que su suma es menor que uno $(\delta s+\delta d<1$, ya que de otro modo el rotor tocaría el estator). En este ejemplo se adopta una variación de " $\delta 0$ " del $\pm 10 \%$. La matriz bobinado (ec. 4) multiplicada por la matriz corriente (ec. 5) determina una matriz de fuerza magnetomotriz estatórica (máquina girando en vacío) determinando un flujo de entrehierro para la corriente de vacío (ec. 6). Observando la ecuación (15) se ve que cada columna de la matriz $\mathbf{P}\left(\chi \times \omega t_{n}\right)=\left[\mathbf{p}_{1} \cdots \mathbf{p}_{\omega t_{n}}\right]$ define el valor de la permeancia para cada posición angular fija del rotor en cada instante de tiempo, donde cada elemento " $\mathbf{P}_{\mathrm{ij}}$ " varía alrededor de un valor medio. El valor medio de la permeancia será $1,627 \times 10^{-4}$ [Hy] alcanzando en el entrehierro de la máquina un valor máximo de $1,716 \times 10^{-4}$ y mínimo de $1,533 \times 10^{-4}$ [Hy]. El flujo (ec. 6) presentará por lo tanto una modulación dada por la ecuación (15) y determinará una modificación de los autovalores y los autovectores como se ve en las Figuras (4) $y(5)$.

Para la máquina sin falla (Fig. 3) se obtienen dos autovalores $(0,09$ y 0,091) siendo la variación en falla muy pequeña del orden de $10^{-7}$ (Fig. 5). Sin embargo, los dos autovectores para la máquina sin falla (Fig. 4) difieren de los autovectores en la máquina eléctrica en falla (Fig. 6) en magnitud y fase. Cuando la falla es incipiente y los valores son de muy bajo valor, es importante incluir en el análisis los autovectores de los autovalores existentes. Estos muestran una variación tanto en dirección como en magnitud, considerando que ello muestra una variación en la configuración espacial de los enlaces magnéticos. 


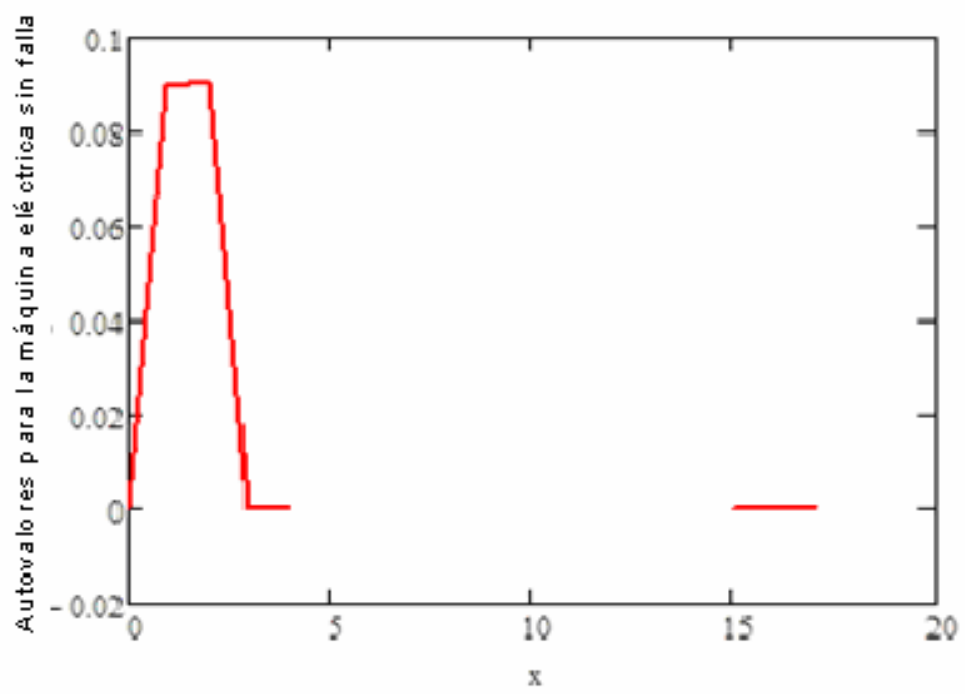

Fig. 3: Autovalores para la máquina eléctrica sin falla.

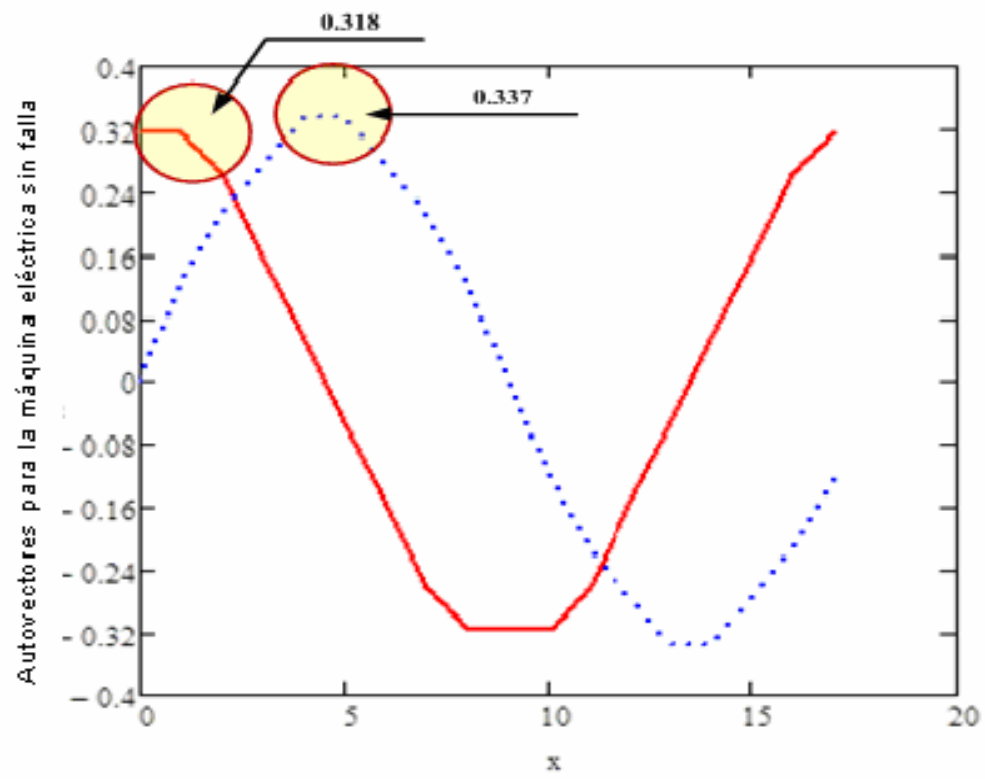

Fig. 4: Autovectores para la máquina eléctrica sin falla.

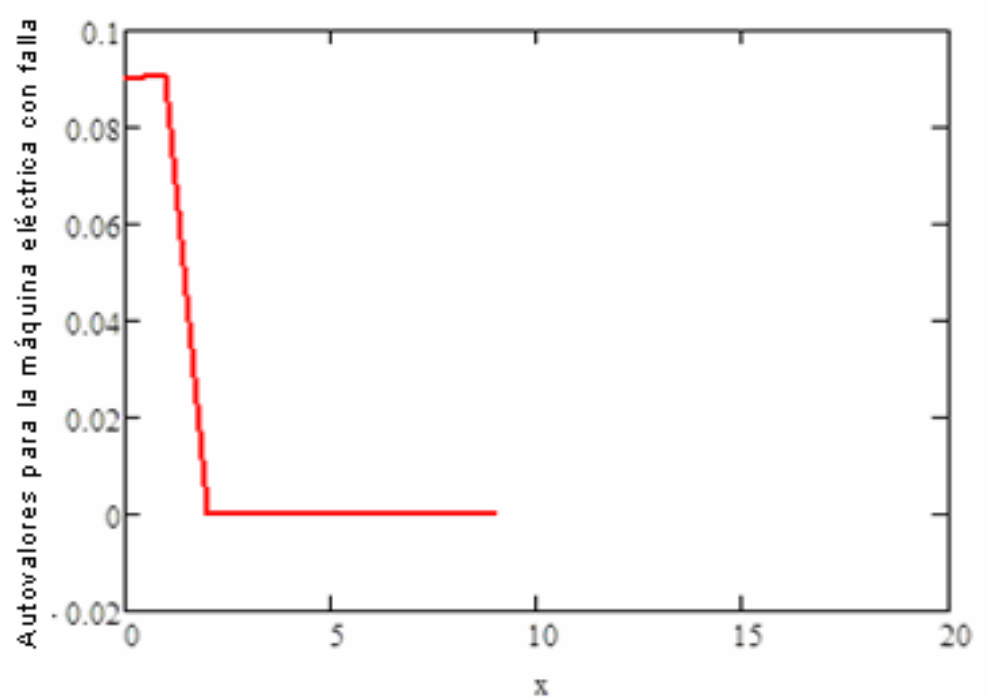

Fig. 5: Autovalores para la máquina eléctrica con falla. 


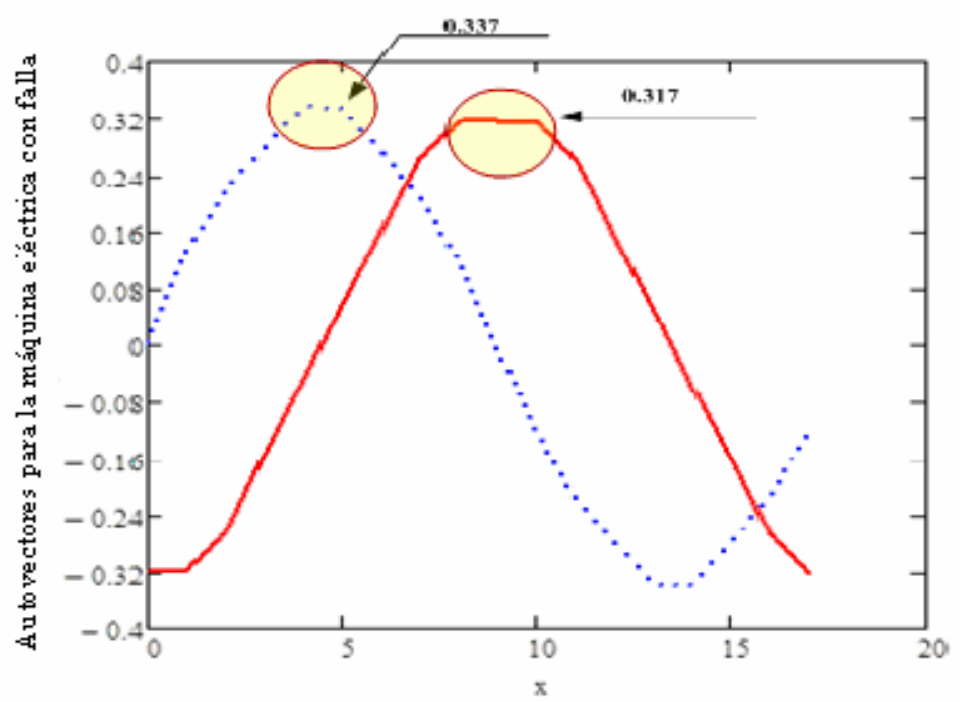

Fig. 6: Autovectores para la máquina eléctrica con falla.

\section{CONCLUSIONES}

Se destaca el análisis matemático previo como una propuesta novedosa que admite expresar las estructuras en forma matricial. De estas expresiones surge la posibilidad de medición del flujo disperso sobre la máquina eléctrica, permitiendo su disposición matricial y posterior análisis por autovalores y por determinación espectral, aplicando la Transformada Rápida de Fourier. Considerando que el autovalor representa una variación de la magnitud de enlace magnético y el autovector una modificación en la dirección y sentido de los enlaces, las variaciones de ambos permitirían orientar el diagnóstico hacia el tipo de falla, y ciertamente como abarca la totalidad de las fallas puede augurarse que tal vez se convierta en un método de diagnóstico. Esta afirmación no impide considerar que el método permite determinar la frecuencia de falla y por lo tanto diagnosticar la misma (también en este caso todas las fallas: eléctricas y mecánicas) (di Prátula, 2009; di Prátula y Russin, 2010).

Los ensayos preliminares con sensores (bobinas con núcleo de aire) muestran resultados halagüeños para su aplicación experimental, ya que los sensores no superan el costo de U\$S 20 cada uno, y se requiere de la aplicación de un osciloscopio industrial para determinar frecuencias y datos de la onda de flujo. Para la medición del flujo se colocarían: tres sensores a lo largo del eje de la máquina eléctrica (axial) en cuatro lugares sobre la máquina (repartidas sobre la parte superior) y posteriormente (también simultáneamente) cuatro sensores en tres posiciones (repartidas axialmente). Las posiciones adoptadas obedecen a la idea de captar la señal sobre la ranura a lo largo (un valor instantáneo de flujo) y sobre varias ranuras (variación de flujo en el tiempo). Finalmente, una ventaja importante es que puede usarse para monitoreo continuo o para medición periódica.

\section{REFERENCIAS}

Al-Nuaim, N.A. y H.A. Toliyat, A Novel Method for Modeling Dynamic Air-Gap Eccentricity in Synchronous Machines Based on Modified Winding Function Theory, IEEE Transactions on Energy Conversion, 13(2):156-162 (1998).

Amirat, Y., M.E.H. Benbouzid, B. Bensaker y R. Wamkeue, Condition Monitoring and Fault Diagnosis in Wind Energy Conversion Systems: A Review, IEEE International Conference on Electric Machines \& Drives, 2:1434-1439 (2007).

Andrawus, J.A., Maintenance optimization for Wind Turbines, Thesis for the degree of Doctor of Philosophy, Registration No. SCO13781, The Robert Gordon University, Scotland (2008).

Bambill, E., Aproximación general para calcular operadores inductivos del trabajo de las máquinas eléctricas, por discretización de su correspondiente recinto magnético, Revista Electrotecnia №2, pp. 43-53, Marzo/Abril (1989). 
di Prátula, H.R., New proposals for outside measuring and analyzing the failure in the induction machine, Electric Machines and Drives Conference IEMDC'03, 2(2):917- 922 (2003a).

di Prátula, H.R., Una visión holística de la máquina de inducción y análisis estructural en falla con medición no invasiva de las variables eléctricas y magnéticas, Conferencia XRPIC, Vol.1, 165-170, San Nicolás, Argentina, 8 al 10 de Octubre (2003b).

di Prátula, H.R., Diagnóstico Teórico y Experimental de Fallas Rotóricas en Máquinas Eléctricas de Inducción, Tesis Doctoral, Número de Registro 27476, Expediente D-2625/98, Universidad Nacional del Sur, Bahía Blanca, Argentina (2008).

di Prátula, H.R., Diagnóstico predictivo de fallas rotóricas en máquinas eléctricas de inducción, Revista Digital del Instituto Universitario Naval (en línea, fecha de consulta Mayo 12, 2011; www.inun.edu.ar/menues/investigacion/revista/REVISTA DIGITAL DEL INUN.pdf), ISSN 18527205, Año 1, No. 1, pp. 79-89 (2009)

di Prátula, H.R. y A. Russin, Mantenimiento predictivo de turbinas eólicas, I Jornadas de Ciencia y Tecnología del Instituto Universitario Naval, ISSN 1853-1377 (en línea) pp.127-133 (fecha de consulta Mayo 12, 2011; http://www.ara.mil.ar/archivos/Docs/13-jornadas del cyt.pdf), Buenos Aires, Argentina, 26 y 27 de Agosto (2010).

Dorrell, D.G., W.T Thomson y S. Roach, Analysis of airgap flux, current, and vibration signals as a function of the combination of static and dynamic airgap eccentricity in 3-phase induction motors, IEEE Transactions on Industry Applications, 33(1):24-34 (1997).

Fernandez Cabanas, M., M.G. Melero, G.A. Orcajo, J.M. Cano Rodriguez y J.S. Sariego, Técnicas para el mantenimiento y diagnóstico de máquinas eléctricas rotativas, Ed. Marcombo, ABB Service, ISBN 84-267-1166-9 (1998).

Henao, H., C. Demian y G.A. Capolino, A Frequency-Domain Detection of Stator Winding Faults in Induction Machines Using an External Flux Sensor, Industry Applications, IEEE Transactions, 39(5):1272-1279 (2003).

Henderson, A.R., M. Gleeson, U. Kaufmes, J. Jacquemin y C.A. Morgan, Offshore wind due diligence- how country, and life-cycle stage, impacts what to look for, European Wind Energy Conference (en línea, fecha de consulta Mayo 12, 2011;

http://www.gl-garradhassan.com/assets/technical/EWEC2009_168_Due_Diligence__ARHenderson_-_Poster_and_Paper.pdf), Marsella, Francia, 16 al 19 de Marzo (20009).

Riley, P.C.M., B.K. Lin, B.K., T.G. Habetler y R.R. Schoen, A method for sensorless on-line vibration monitoring of induction machines, Proceedings of the IEEE-IAS Annual Meeting, 201-207 (1997).

Schoen, R.R., B.K. Lin, T.G. Habetler, J.H. Schlag y S. Farag, An unsupervised, on-line system for induction motor fault detection using stator current monitoring, Industry Applications, IEEE Transactions, 31(6):1280-1286 (1995).

Serra, F., G. Catuogno, C. De Angelo y G. Bossio, Sistemas de Monitoreo de condición en turbinas eólicas, Avances en Energías Renovables y Medio Ambiente, Vol. 12, pp. 06.57-06.63 (2008).

Verbruggen, T.W., Wind Turbine Operation \& Maintenance based on Condition Monitoring WT- $\Omega$, Final Tech. Report, ECN-C-03-047 (en línea) 2003 (fecha de consulta Mayo 12, 2011; http://www.ecn.nl/docs/library/report/2003/c03047.pdf).

Walford, C.A., Wind Turbine Reliability: Understanding and Minimizing Wind Turbine Operation and Maintenance Costs, SANDIA REPORT SAND2006-1100 (en línea), 2006. (fecha de consulta Mayo 12, 2011; http://prod.sandia.gov/techlib/access-control.cgi/2006/061100.pdf)

Xiaogang, L., L. Yuefeng, H.A. Toliyat, A. El-Antably y T.A. Lipo, Multiple Coupled Circuit Modeling of Induction Machines, IEEE Transactions on Industry Aplications, 31(2):203-210 (1995). 